\title{
Is Central Nervous System an Immune-Privileged Site?
}

\author{
Shrestha R, ${ }^{1,2,5}$ Millington $0,{ }^{3}$ Brewer J, ${ }^{4}$ Bushell $T^{5}$
}

${ }^{1}$ Department of Pharmacology

Dhulikhel Hospital-Kathmandu University Hospital

Kathmandu University School of Medical Sciences Dhulikhel, Kavre, Nepal

${ }^{2}$ Lilly Research Centre, Eli Lilly and Company, Surrey, UK

${ }^{3}$ Centre of Biophotonics, Strathclyde Institute of Pharmacy and Biomedical Sciences, University of Strathclyde, Glasgow, UK

${ }^{4}$ Institute of Infection, Immunity and Inflammation College of Medical, Veterinary and Life Sciences,

University of Glasgow, Glasgow, UK

${ }^{5}$ Centre for Neuroscience, Strathclyde Institute of Pharmacy and Biomedical Sciences, University of Strathclyde, Glasgow, UK

\section{Corresponding Author}

Rajeev Shrestha

Department of Pharmacology

Dhulikhel Hospital-Kathmandu University Hospital

Kathmandu University School of Medical Sciences Dhulikhel, kavre, Nepal

E-mail: rmaleku@hotmail.com

\section{Citation}

Shrestha R, Millington O, Brewer J, Bushell T. Is Central Nervous System an Immune-Privileged Site? Kathmandu Univ Med J 2013;41(1):102-107.

\section{INTRODUCTION}

The central nervous system (CNS) is a complex organ system consisting of billions of neurons that relay information via many billions of synapses. The immense capacity and ability to remodel itself in response to experience and the environment always fascinate neuroscientists, who believe that this organ is the most complicated machine ever known to the universe. The ability to receive, retain, recall and analyze complex information is a wonderful capacity of the nervous system. ${ }^{1}$

Whether or not the CNS is an immune-privileged site has been an intense debate among neuroscientists. The concept of an immuno-privileged site was first highlighted when allografts in eye and brain tissue were better persisted in comparison to other peripheral tissues. ${ }^{2,3}$ These studies demonstrated that the graft showed little rejection in comparison to other parts of the body. On the other hand, proper neuronal function demands a tight regulation of the CNS microenvironment including concentration of different ions and other noxious substances. ${ }^{4}$ For this proper control and the normal function of the CNS, the blood brain barrier (BBB) plays an important role. ${ }^{5}$

The BBB lies at the interface of the blood and the brain tissue that comprises microvascular endothelium, astrocytes, basement-membrane and pericytes. ${ }^{6,7}$ To address the collective terms for the constituents of the $B B B$, the concept of a 'neurovascular unit' was proposed to emphasize more for the structure and function of $B B B$ 
integrity. ${ }^{4,5}$ This unit protects the CNS from fluctuations of nutrients, hormones, metabolites and blood constituents including endogenous and exogenous compounds. ${ }^{4,8}$ The endothelial cells of the BBB have distinct morphological and physiological properties in comparison to other endothelial cells of the body. ${ }^{5,6,9}$ These endothelial cells lack fenestration with a tight junction reducing permeability to intercellular pathways and they express very low numbers of non-specific transporters. ${ }^{6,9,10}$ Moreover, the highly polarized receptors and transporter systems play an important role in the movement of nutrients into the CNS and the efflux of metabolites or toxic substances into the vascular compartment. ${ }^{5,10,11}$ Furthermore, it has also been suggested that pericytes play a major role in maintaining the BBB tight junction along with these endothelial cells. ${ }^{12,13}$ An in vivo imaging study of mice lacking pericytes showed an increase in the permeability of the BBB to water as well as low-molecular and high-molecular mass tracers. ${ }^{12}$

Hence, the presence of a neurovascular unit, lack of conventional lymphatic vessels and limited parenchymal expression of major histocompatibility complex (MHC) molecules in the CNS along with the limited rejection of allografts in the CNS might have led us to consider the CNS as a immunologically privileged area. ${ }^{14}$ However, it is now well accepted that the brain can no longer be considered immune-privileged. There is increasing evidence for the regular communication between the CNS and the immune system. ${ }^{15-17}$ Research has shown that immune cells can enter the CNS via the choroid-plexus and reside in perivascular spaces or move into the neuropil. ${ }^{18-21}$ Furthermore, it has been shown that the extravasation of these immune cells into the CNS involves a very complex set of adhesion molecules present on the endothelial cells and on the cell surface of these immune cells. ${ }^{20-23}$ Common adhesion molecules that have been suggested to be involved in the extravasation of leukocytes include the intracellular adhesion molecule (ICAM) family, vascular cell adhesion molecule (VCAM), integrins, selectins and the junctional adhesion molecule (JAM) family.,14,24-26 Hence, this evidence suggests that the CNS is not an immunologically privileged area. Nevertheless, it is also true that the CNS is a more immunologically privileged site relative to other organs due to the presence of the BBB and tightly regulated immune response including limited movement of immune cells and expression of death ligand. 5,27

Communication between central nervous system and immune system

\section{Brain modulation of immune function}

The most common way of communication is via humoral factors such as cortisol and epinephrine released from the hypothalamic-pituitary-adrenal (HPA) axis in response to stress with a stressor being anything that stimulates the activation of HPA axis and sympathetic nervous system. ${ }^{17,28-30}$ Stress can alter antibody secretions as well as the release of pro-inflammatory as well as anti-inflammatory mediators. ${ }^{31}$ Most immune cells express receptors for one or more hormones associated with HPA and sympatheticadrenal-medullary axis and these hormones can regulate immune function via these receptors such as inhibition of inflammation and shifting production of cytokines from Th- 1 cells to Th- 2 cells, trafficking cells from lymph node to peripheral blood, increasing cytokine production and maintaining competence of immune cells including $T$ cells, B cells and macrophages..$^{29,32-36}$ It has also been demonstrated that mice fail to recover from experimental autoimmune encephalomyelitis (EAE) after adrenalectomy , which suggests that the HPA axis plays a crucial role in modulation of the immune system as inflammation is known to be critically involved in the progression of this animal model of Multiple sclerosis (MS). ${ }^{37}$ Moreover, nerve fibres present in the lymphoid organs also suggest a bidirectional communication between the CNS and immune system. Recently, it has been demonstrated that stimulation of vagus nerve fibres causes acetylcholine release from spleenic T cells. ${ }^{38}$ These nerve fibres act as a link between these two systems which relay or receive signals and affect the physiological functions (such as antibody secretion and cytokines and chemokines release) of the immune cells. ${ }^{38-40}$

\section{Immune regulation of brain function}

Whilst it is clear that the CNS can influence immunity, the immune system also plays a crucial role in normal brain development, neuronal differentiation and synaptic plasticity. ${ }^{41,42}$ Furthermore, various cytokines and chemokines are able to regulate secretion of neurotransmitters in the CNS, with interferons being the first cytokines identified to have an effect on neuronal function. .8,43-47 $^{2}$ Cytokines can induce sickness behaviour (characterised by fever, reduction in activity and weight loss) and also regulate neuroendocrine effects, including release of different hormones. ${ }^{48,49}$ Moreover, cytokines including interleukin (IL)-1, IL-2 and IL-6 are also found to regulate neuronal functions. ${ }^{28,39,46} \mathrm{IL}-1$ and IL-6 stimulate HPA axis in secretion of corticotrophin-releasing hormones, whereas IL-2 can act as a neuroregulatory cytokine in the CNS. ${ }^{28,46,50,51}$ IL-2 can affect the growth and survival of neurites, proliferation and maturation of oligodendrocytes, growth of microglia and also has effect on behaviour and electrocorticogram spectrum..$^{52-55}$ It has also been shown that systemic immunisation in mice caused a decrease in neurotransmitter levels in specific regions of the brain such as hypothalamus, hippocampus, locus coeruleus, supraoptic nucleus and paraventricular nucleus while causing an increase in the nucleus tractus solitarius. ${ }^{44,56,57}$ From these studies, it is evident that the CNS and the immune system communicate with each other via various mediators. Furthermore, there are also several studies showing a direct interaction between cells of the CNS and cells of the immune system. ${ }^{58-60}$ These immune cells are regularly patrolling the CNS and scanning for their cognate antigens in healthy as well as in diseased models like EAE. ${ }^{19,24,61,62}$ Details of their movement and functions are dealt with in the sections below. 


\section{Movement of lymphocytes in and out of the CNS}

As discussed above, there is a continuous communication between the CNS and the immune system either via various neuromediators / immunomediators or direct cell to cell interactions. In direct cell to cell interactions, immune cells especially leukocytes migrate into the CNS. Three pathways have been proposed for leukocytes movement to and from the CNS: i) from blood to cerebrospinal fluid (CSF) across the choroids plexus (the site of the blood-CSF barrier), ii) from blood to subarachnoid space at the pial surface of the brain and iii) from blood to parenchymal perivascular space. ${ }^{63}$ The first two pathways seem to be the most relevant under normal physiological conditions [64] whereas extravasation by the third pathway occurs only during an inflammatory event. ${ }^{19,65-67}$ Normally, lymphocytes (predominantly $\mathrm{T}$ lymphocytes) migrate through the fenestrated endothelium of the choroid plexus stroma, interact with epithelial cells of the choroid plexus and enter the CSF at its site of formation. ${ }^{63,68,69}$ It is known that activated $T$ cells express specific chemokine receptors which enable them to interact with adhesion molecules expressed in the vascular endothelium. ${ }^{14,70,71}$ In this process, only activated lymphocytes can undergo this extravasation event, independent of antigen specificity as these cells express chemokine receptors which help in this extravasation. . $^{14,20,22,25,72}$ This process of extravasation requires defined interactions between molecules expressed in lymphocytes and endothelial cells of the BBB. These discrete steps include interaction between selectins and their ligands, integrins and cell adhesion molecules (CAMs), chemokines and their receptors, matrix metalloproteinase (MMPs) and their tissue inhibitors (TIMPs).

The initial contact between a leukocyte and an endothelial cell is referred to as 'tethering' and subsequent interactions are referred to as 'rolling' occur in the peripheral high endothelial venules (Fig 1A). These initial events of leukocyte recruitment are primarily mediated by the interaction between P-selectin glycoprotein ligand-1 (PSGL1 ; expressed by all lymphocytes) and selectins. However, the role of endothelial P-selectin in recruiting lymphocytes into the CNS is still controversial. Although, some studies have suggested that $\mathrm{P}$-selectin has a crucial role in the early migration of lymphocytes into the non-inflamed brain, others have suggested that there is no role of P-selectin in the migration of lymphocytes during inflammatory condition within CNS since P-selectin deficient mice also develop EAE which is indistinguishable from wildtype. ${ }^{64,68,73,74,76-78}$ The 'rolling' of lymphocytes is followed by the interaction of $\alpha 4 \beta 1$ molecule ( $\alpha 4$-integrin) expressed in the membrane of lymphocytes with vascular cell adhesion molecule (VCAM)-1 expressed on the endothelial surface which causes the capture of these lymphocytes. ${ }^{23,79}$ This capturing process allows lymphocytes a sufficient time to interact with chemokine ligands (CCL19 and CCL21) expressed on the endothelial surface and leads to the activation as well as migration of lymphocytes into the

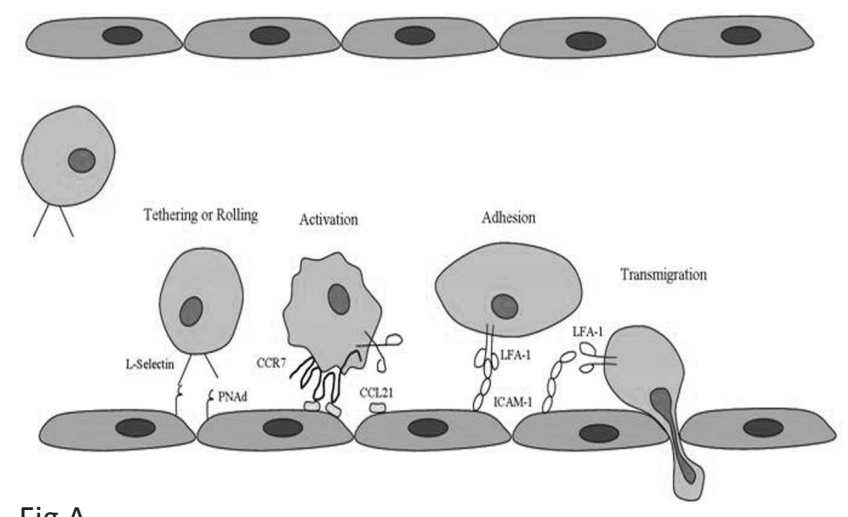

Fig A
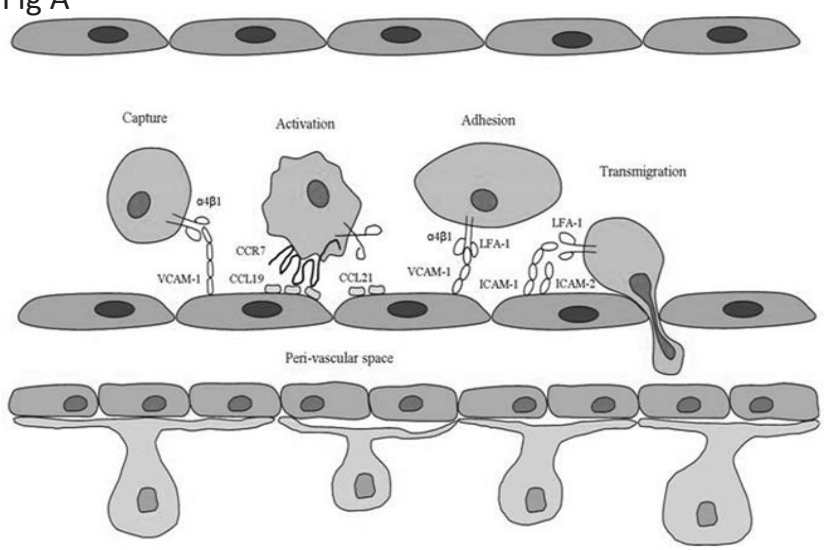

Fig B

Figure 1. Schematic showing the multistep model of lymphcytes movement in two different compartments. (A) In the peripheral vascular endothelium, the extravasation of lymphocyte starts with tethering or rolling followed by activation, adhesion and transmigration.(B) At the endothelium of spinal cord, the extravasation of lymphocyte starts with direct capture without tethering or rolling and followed by activation, adhesion and migration into perivascular space. (Adapted from Engelhardt, 2006).

brain and also causes them to adhere on the endothelial surface more firmly. ${ }^{80}$ At this stage lymphocyte functionassociated antigen (LFA)-1 expressed on the membrane of lymphocytes, binds with intercellular adhesion molecule (ICAM)-1 or ICAM-2 expressed on the endothelial surface causing transendothelial migration of lymphocytes. The interaction between LFA-1 and ICAM-1 or ICAM-2 is considered to be crucial in the transendothelial migration of lymphocytes into the perivascular spaces of the CNS as it has been demonstrated that lack of ICAM-1 and ICAM- 2 in mice fails to recruit lymphocytes into the CNS. ${ }^{81}$ Moreover, it is important to note that lymphocyte recruitment in peripheral vascular endothelium is different than their recruitment into the perivascular area of the CNS. In the peripheral vascular endothelium, as explained above, there is a 'tethering' and 'rolling' of lymphocytes on the endothelial surface which is absent in vascular endothelium of the BBB (Fig 1B)..$^{82}$ In the vascular endothelium of the $B B B$, there is immediate capture of the lymphocytes (predominantly $\mathrm{T}$ cells) followed by activation, adhesion and transmigration. ${ }^{14}$

Lymphocytes patrol the CNS regularly and scan for their cognate antigens in healthy as well as the inflamed 
CNS.$^{19,22,61}$ It has also been demonstrated that during a strong systemic immune response, there is an increase in lymphocytes (predominantly T cells) number in the CNS. ${ }^{83}$ Those $\mathrm{T}$ cells which recognise their cognate antigens/MHC complex initiate an immune reaction and reside in the CNS while those failing to recognise cognate antigens in the context of MHC molecules exit from the CNS suggesting that the entry of T cells into the CNS is dependent on their activation state rather than antigen specificity. ${ }^{22,65,84-87}$

The interaction between lymphocytes and cells of the CNS and antigen presentation usually occurs in the perivascular spaces of the brain known as 'Virchow-Robin' spaces. ${ }^{22,63,88,89}$ As described previously, the initial entry of lymphocytes into the brain is suggested to be via the subarachnoid space, and/or the blood-CSF barrier, where selectins and adhesion molecules are expressed.63 Antigen presentation occurs between lymphocytes and microglial cells and also with astrocytes during neurodegenerative disease including multiple sclerosis. ${ }^{90-93}$ Several studies

\section{REFERENCES}

1. Weil ZM, Norman GJ, DeVries AC, Nelson RJ. The injured nervous system: a Darwinian perspective. Prog Neurobiol 2008; 86(1): 48-59.

2. Barker CF, Billingham RE. Immunologically privileged sites. Adv Immunol 1977; 25: 1-54.

3. Billingham RE, Boswell T. Studies on the problem of corneal homografts. Proc R Soc Lond B Biol Sci 1953; 141(904): 392-406.

4. Hawkins BT, Davis TP. The Blood-Brain Barrier/Neurovascular Unit in Health and Disease. Pharmacological Reviews 2005; 57(2): 173-185.

5. Weiss N, Miller F, Cazaubon S, Couraud PO. The blood-brain barrier in brain homeostasis and neurological diseases. Biochim Biophys Acta 2009;1788(4): 842-57.

6. Persidsky Y, Ramirez SH, Haorah J, Kanmogne GD. Blood-brain barrier: structural components and function under physiologic and pathologic conditions. J Neuroimmune Pharmacol 2006; 1(3): 223-36.

7. Wolburg H, Lippoldt A. Tight junctions of the blood-brain barrier: development, composition and regulation. Vascul Pharmacol 2002;38(6): 323-37.

8. Bernacki J, Dobrowolska A, Nierwinska K, Malecki A. Physiology and pharmacological role of the blood-brain barrier. Pharmacol Rep 2008; 60(5): 600-22.

9. Abbott N. Dynamics of CNS Barriers: Evolution, Differentiation, and Modulation. Cellular and Molecular Neurobiology 2005; 25(1): 5-23.

10. Petty MA, Lo EH. Junctional complexes of the blood-brain barrier: permeability changes in neuroinflammation. Prog Neurobiol 2002;68(5): 311-23.

11. Brightman MW, Kadota Y. Nonpermeable and permeable vessels of the brain. NIDA Res Monogr 1992; 120: 87-107.

12. Armulik A. Pericytes regulate the blood-brain barrier. Nature 2010; 468(7323):557-561.

13. Balabanov R, Dore-Duffy P. Role of the CNS microvascular pericyte in the blood-brain barrier. Journal of Neuroscience Research 1998;53(6): 637-644.

14. Engelhardt B. Molecular mechanisms involved in $T$ cell migration across the blood-brain barrier. J Neural Transm 2006;113(4): 477-85.

15. Bucky Jones T, Lucin KM, Popovich PG, Adriana del Rey GPC, Hugo $\mathrm{OB}$. The Immune System of the Brain, in Neurolmmune Biology. Elsevier;2007p.127-144. have confirmed that microglial cells can express MHCII protein and are effective antigen presenting cells. ${ }^{90,91}$ Similarly, astrocytes also express MHC-II proteins and can act as antigen presenting cells. ${ }^{92,93}$ Along with these cells, dendritic cells and macrophages, the professional antigen presenting cells, also help in antigen presentation to lymphocytes in the perivascular space. ${ }^{90}$ Alternatively, it has also been shown that neurons interact with $\mathrm{T}$ cells via B7 ligand in the absence of MHC-II molecules as they do not express MHC-II molecules. ${ }^{60}$

Hence, from all these studies, it is evident that communication exists between the CNS and the immune system. These two systems are interacting via various soluble factors as well as via direct cell to cell contact. Further, it is also apparent that these two systems are not working independently or are autonomous but working together to maintain proper physiological functions. However, the precise role of immune system in healthy and diseased state of the CNS is still under investigation.

16. Kerschensteiner M, Meinl E, Hohlfeld R. Neuro-immune crosstalk in CNS diseases. Neuroscience 2009; 158(3): 1122-32.

17. Straub RH, Westermann J, Scholmerich J, Falk W. Dialogue between the CNS and the immune system in lymphoid organs. Immunol Today 1998;19(9): 409-13.

18. Carson MJ, Doose JM, Melchior B, Schmid CD, Ploix CC. CNS immune privilege: hiding in plain sight. Immunological Reviews 2006; 213(1): 48-65.

19. Hickey WF. Migration of hematogenous cells through the blood-brain barrier and the initiation of CNS inflammation. Brain Pathol 1991; 1(2):97-105.

20. Reboldi A. C-C chemokine receptor 6-regulated entry of $\mathrm{TH}-17$ cells into the CNS through the choroid plexus is required for the initiation of EAE. Nat Immunol 2009;10(5):514-523.

21. Weller RO, Engelhardt B, Phillips MJ. Lymphocyte Targeting of the Central Nervous System: A Review of Afferent and Efferent CNSImmune Pathways. Brain Pathology 1996;6(3):275-288.

22. Hickey WF, Hsu BL, Kimura H. T-lymphocyte entry into the central nervous system. J Neurosci Res 1991;28(2): 254-60.

23. Laschinger M, Engelhardt B. Interaction of [alpha]4-integrin with VCAM-1 is involved in adhesion of encephalitogenic T cell blasts to brain endothelium but not in their transendothelial migration in vitro. Journal of Neuroimmunology 2000;102(1):32-43.

24. Engelhardt B. T cell migration into the central nervous system during health and disease: Different molecular keys allow access to different central nervous system compartments. Clinical and Experimental Neuroimmunology 2010;(2):79-93.

25. Engelhardt B, Wolburg H. Mini-review: Transendothelial migration of leukocytes: through the front door or around the side of the house? Eur J Immunol 2004;34(11):2955-63.

26. Springer TA. Traffic signals for lymphocyte recirculation and leukocyte emigration: the multistep paradigm. Cell 1994;76(2):301-14.

27. Galea I, Bechmann I, Perry VH. What is immune privilege (not)? Trends in Immunology 2007;28(1): 12-18.

28. Blalock JE. The syntax of immune-neuroendocrine communication. Immunology Today 1994;5(11):504-511.

29. Glaser R, Kiecolt-Glaser JK. Stress-induced immune dysfunction: implications for health. Nat Rev Immunol 2005;5(3):243-251. 
30. Segerstrom SC, Miller GE. Psychological Stress and the Human Immune System: A Meta-Analytic Study of 30 Years of Inquiry. Psychological Bulletin 2004;130(4):601-630.

31. Konstantinos AP, Sheridan JF. Stress and influenza viral infection: modulation of proinflammatory cytokine responses in the lung. Respiration Physiology 2001;128(1): 71-77.

32. Miller AH. Glucocorticoid Receptors Are Differentially Expressed in the Cells and Tissues of the Immune System. Cellular Immunology 1998;186(1): 45-54

33. Welniak LA, Sun R, Murphy WJ. The role of growth hormone in T-cell development and reconstitution. Journal of Leukocyte Biology 2002;71(3): 381-387.

34. Elenkov IJ, Chrousos GP. Stress Hormones, Proinflammatory and Antiinflammatory Cytokines, and Autoimmunity. Annals of the New York Academy of Sciences 2002;966(1):290-303.

35. Lai J-P, Douglas SD, Ho W-Z. Human lymphocytes express substance $P$ and its receptor. Journal of Neuroimmunology 1998;86(1):80-86.

36. Radulovic M, Dautzenberg FM, Sydow S, Radulovic J, Spiess J. Corticotropin-Releasing Factor Receptor 1 in Mouse Spleen: Expression After Immune Stimulation and Identification of ReceptorBearing Cells. The Journal of Immunology 1999;162(5):3013-3021.

37. MacPhee IA, Antoni FA, Mason DW. Spontaneous recovery of rats from experimental allergic encephalomyelitis is dependent on regulation of the immune system by endogenous adrenal corticosteroids. The Journal of Experimental Medicine 1989;169(2):431-445.

38. Rosas-Ballina M. Acetylcholine-Synthesizing T Cells Relay Neural Signals in a Vagus Nerve Circuit. Science 2011;334(6052):98-101.

39. Madden KS, Felten DL. Experimental basis for neural-immune interactions. Physiological Reviews 1995;75(1):77-106.

40. Felten DL. Noradrenergic sympathetic neural interactions with the immune system: structure and function. Immunol Rev 1987;100: 225-60.

41. Boulanger LM, Shatz CJ. Immune signalling in neural development, synaptic plasticity and disease. Nat Rev Neurosci 2004;5(7):521-531.

42. Deverman BE, Patterson PH. Cytokines and CNS Development. Neuron 2009;64(1):61-78.

43. Blalock JE, Smith EM. Human leukocyte interferon: structural and biological relatedness to adrenocorticotropic hormone and endorphins. Proc Natl Acad Sci U S A 1980;77(10):5972-4.

44. Carlson SL, Felten DL, Livnat S, Felten SY. Alterations of monoamines in specific central autonomic nuclei following immunization in mice. Brain, Behavior, and Immunity 1987;1(1): 52-63.

45. DantzerR.Innateimmunityattheforefrontofpsychoneuroimmunology. Brain, Behavior, and Immunity 2004;18(1):1-6.

46. Jiang C-L, Lu C-L, Liu X-Y. The molecular basis for bidirectional communication between the immune and neuroendocrine systems. Domestic Animal Endocrinology 1998;15(5):363-369.

47. Smith EM, Blalock JE. Human lymphocyte production of corticotropin and endorphin-like substances: association with leukocyte interferon. Proc Natl Acad Sci U S A 1981;78(12):7530-4.

48. Crane JW, Buller KM, Day TA. Evidence that the bed nucleus of the stria terminalis contributes to the modulation of hypophysiotropic corticotropin-releasing factor cell responses to systemic interleukin13. The Journal of Comparative Neurology 2003;467(2): 232-242.

49. Schiepers OJG, Wichers MC, Maes M. Cytokines and major depression. Progress in Neuro-Psychopharmacology and Biological Psychiatry 2005;29(2): 201-217.

50. McCann SM. Role of Cytokines in the Endocrine Systema. Annals of the New York Academy of Sciences 1994;741(1):50-63.

51. Hanisch U-K, Quirion R. Interleukin-2 as a neuroregulatory cytokine. Brain Research Reviews 1995;21(3):246-284.
52. Sarder M, Saito H, Abe K. Interleukin-2 promotes survival and neurite extension of cultured neurons from fetal rat brain. Brain Research 1993;625(2):347-350.

53. Benveniste EN, Merrill JE. Stimulation of oligodendroglial proliferation and maturation by interleukin-2. Nature 1986;321(6070): 610-613.

54. Sawada M, Suzumura A, Marunouchi T. Induction of Functional Interleukin-2 Receptor in Mouse Microglia. Journal of Neurochemistry 1995;64(5):1973-1979.

55. De Sarro GB, Masuda Y, Ascioti C, Audino MG, Nistico G. Behavioural and ECoG spectrum changes induced by intracerebral infusion of interferons and interleukin 2 in rats are antagonized by naloxone. Neuropharmacology 1990; 29(2):167-179.

56. Besedovsky $\mathrm{H}$. The immune response evokes changes in brain noradrenergic neurons. Science 1983;221(4610):564-566.

57. Zalcman S, Shanks N, Anisman H. Time-dependent variations of central norepinephrine and dopamine following antigen administration. Brain Research 1991;557(1-2):69-76.

58. Aloisi F, Ria F, Penna G, Adorini L. Microglia are more efficient than astrocytes in antigen processing and in Th1 but not Th2 cell activation. $\mathrm{J}$ Immunol 1998;160(10):4671-80.

59. Greter M. Dendritic cells permit immune invasion of the CNS in an animal model of multiple sclerosis. Nat Med 2005;11(3):328-334.

60. Liu Y, Teige I, Birnir B, Issazadeh-Navikas S. Neuron-mediated generation of regulatory $T$ cells from encephalitogenic $T$ cells suppresses EAE. Nat Med 2006;12(5):518-25.

61. Engelhardt B, Ransohoff RM. The ins and outs of T-lymphocyte trafficking to the CNS: anatomical sites and molecular mechanisms. Trends Immunol 2005;26(9): 485-95.

62. Lampson LA. Enhanced T Cell Migration to Sites of Microscopic CNS Disease: Complementary Treatments Evaluated by 2- and 3-D Image Analysis. Brain Pathology 1994;4(2):125-134.

63. Ransohoff RM, Kivisakk P, Kidd G. Three or more routes for leukocyte migration into the central nervous system. Nat Rev Immunol 2003; 3(7):569-81.

64. Carrithers MD, Visintin I, Viret C, Janeway CA. Role of genetic background in $\mathrm{P}$ selectin-dependent immune surveillance of the central nervous system. Journal of Neuroimmunology 2002;129(1-2): 51-57.

65. Bartholomaus I. Effector $T$ cell interactions with meningeal vascular structures in nascent autoimmune CNS lesions. Nature 2009;462(7269):94-98.

66. Hickey WF, Kimura H. Perivascular microglial cells of the CNS are bone marrow-derived and present antigen in vivo. Science 1988;239(4837):290-2.

67. Piccio L. Molecular Mechanisms Involved in Lymphocyte Recruitment in Inflamed Brain Microvessels: Critical Roles for P-Selectin Glycoprotein Ligand-1 and Heterotrimeric Gi-Linked Receptors. The Journal of Immunology 2002;168(4):1940-1949.

68. Kivisäkk P. Human cerebrospinal fluid central memory CD4+ T cells: evidence for trafficking through choroid plexus and meninges via P-selectin. Proc Natl Acad Sci U S A 2003;100(14):8389-94.

69. Seabrook TJ, Johnston M, Hay JB. Cerebral spinal fluid lymphocytes are part of the normal recirculating lymphocyte pool. J Neuroimmunol 1998;91(1-2):100-7.

70. Engelhardt B, Conley FK, Butcher EC. Cell adhesion molecules on vessels during inflammation in the mouse central nervous system. Journal of Neuroimmunology 1994;51(2):199-208.

71. Jenkins MK. IN VIVO ACTIVATION OF ANTIGEN-SPECIFIC CD4 T CELLS Annual Review of Immunology 2001. 19(1):23-45.

72. Axtell RC, Steinman L. Gaining entry to an uninflamed brain. Nat Immunol 2009;10(5):453-455. 
73. Kerfoot SM, Kubes P. Overlapping roles of P-selectin and alpha 4 integrin to recruit leukocytes to the central nervous system in experimental autoimmune encephalomyelitis. J Immunol 2002;169(2):1000-6.

74. Engelhardt B. Immune cell entry into the central nervous system: Involvement of adhesion molecules and chemokines. Journal of the Neurological Sciences 2008;274(1-2):23-26.

75. Engelhardt B. P-Selectin Glycoprotein Ligand 1 Is Not Required for the Development of Experimental Autoimmune Encephalomyelitis in SJL and C57BL/6 Mice. The Journal of Immunology 2005;175(2):12671275.

76. Engelhardt B, Vestweber D, Hallmann R, Schulz M. E- and P-Selectin Are Not Involved in the Recruitment of Inflammatory Cells Across the Blood-Brain Barrier in Experimental Autoimmune Encephalomyelitis. Blood 1997;90(11):4459-4472.

77. Döring A, Wild M, Vestweber D, Deutsch U, Engelhardt B. E- and P-Selectin Are Not Required for the Development of Experimental Autoimmune Encephalomyelitis in C57BL/6 and SIL Mice. The Journal of Immunology 2007;179(12):8470-8479.

78. Kerfoot SM. Reevaluation of P-Selectin and $\mathbf{I} \pm 4$ Integrin as Targets for the Treatment of Experimental Autoimmune Encephalomyelitis. The Journal of Immunology 2006;176(10):6225-6234.

79. Vajkoczy $P$, Laschinger M, Engelhardt B. Î \pm -integrin-VCAM-1 binding mediates $G$ proteinâ $€$ "independent capture of encephalitogenic $T$ cell blasts to CNS white matter microvessels. The Journal of Clinical Investigation 2001;108(4):557-565.

80. Alt C, Laschinger M, Engelhardt B. Functional expression of the lymphoid chemokines CCL19 (ELC) and CCL 21 (SLC) at the bloodbrain barrier suggests their involvement in G-protein-dependent lymphocyte recruitment into the central nervous system during experimental autoimmune encephalomyelitis. European Journal of Immunology 2002;32(8): 2133-2144.

81. Lyck R. T-cell interaction with ICAM-1/ICAM-2 double-deficient brain endothelium in vitro: the cytoplasmic tail of endothelial ICAM-1 is necessary for transendothelial migration of T cells. Blood 2003; 102(10):3675-3683.
82. Butcher EC. Lymphocyte Trafficking and Regional Immunity, in Advances in Immunology. Academic Press;1999.p 209-253.

83. Hickey WF, Kimura H. Graft-vs.-host disease elicits expression of class I and class II histocompatibility antigens and the presence of scattered T lymphocytes in rat central nervous system. Proc Natl Acad Sci U S A 1987;84(7):2082-6.

84. Becher B, Bechmann I, Greter M. Antigen presentation in autoimmunity and CNS inflammation: how T lymphocytes recognize the brain. J Mol Med 2006;84(7):532-43.

85. Odoardi F, Kawakami N, Klinkert WEF, Wekerle H, FIÃ $1 / 4$ gel A. Blood-borne soluble protein antigen intensifies $T$ cell activation in autoimmune CNS lesions and exacerbates clinical disease. Proceedings of the National Academy of Sciences 2007;104(47): 18625-18630.

86. Hickey WF. Leukocyte traffic in the central nervous system: the participants and their roles. Semin Immunol 1999;11(2):125-37.

87. Hirschberg DL. Accumulation of passively transferred primed T cells independently of their antigen specificity following central nervous system trauma. J Neuroimmunol 1998;89(1-2):88-96.

88. Archambault AS, Sim J, Gimenez MAT, Russell JH. Defining antigendependent stages of $\mathrm{T}$ cell migration from the blood to the central nervous system parenchyma. European Journal of Immunology 2005; 35(4):1076-1085.

89. Goverman J. Autoimmune $T$ cell responses in the central nervous system. Nat Rev Immunol 2009;9(6):393-407.

90. Becher B, Prat A, Antel JP. Brain-immune connection: Immunoregulatory properties of CNS-resident cells. Glia 2000;29(4): 293-304.

91. Bö L. Detection of MHC class II-antigens on macrophages and microglia, but not on astrocytes and endothelia in active multiple sclerosis lesions. Journal of Neuroimmunology 1994;51(2):135-146.

92. Neumann H. Control of glial immune function by neurons. Glia 2001;36(2):191-9.

93. Zeinstra E, Wilczak N, Streefland C, De Keyser J. Astrocytes in chronic active multiple sclerosis plaques express MHC class II molecules. Neuroreport 2000;11(1): 89-91. 